\title{
Telomerase reverse transcriptase promoter mutations in thyroid carcinomas: implications in precision oncology-a narrative review
}

\author{
Xiaotian Yuan ${ }^{1,2}$, Tiantian Liu ${ }^{1}$, Dawei $\mathrm{Xu}^{2,3} \wedge$ \\ ${ }^{1}$ School of Medicine, Cheeloo College of Medicine, Shandong University, Jinan, China; ${ }^{2}$ Department of Medicine, Division of Hematology, \\ Bioclinicum and Center for Molecular Medicine (CMM), Karolinska Institutet and Karolinska University Hospital Solna, Solna, Sweden; ${ }^{3}$ Karolinska \\ Institute-Shandong University Collaborative Laboratory for Cancer and Stem Cell Research, Jinan, China \\ Contributions: (I) Conception and design: X Yuan, T Liu, D Xu; (II) Administrative support: D Xu; (III) Provision of study materials or patients: \\ None; (IV) Collection and assembly of data: X Yuan, T Liu; (V) Data analysis and interpretation: All authors; (VI) Manuscript writing: All authors; (VII) \\ Final approval of manuscript: All authors. \\ Correspondence to: Dr. Tiantian Liu. School of Medicine, Shandong University, Jinan, China. Email: liu.tiantian@sdu.edu.cn.
}

\begin{abstract}
Telomerase is a ribonucleoprotein enzyme with telomerase reverse transcriptase (TERT) as a catalytic component. In normal human follicular thyroid cells or thyrocytes, telomerase is silent due to the TERT gene being tightly repressed. However, during the formation of thyroid carcinoma (TC), telomerase becomes activated via TERT induction. The TERT promoter's gain-of-function mutation has recently been identified in TCs and many other malignancies. The mutation creates a de novo ETS-binding motif through which TERT transcription is de-repressed and telomerase is activated; through this, the mutant TERT promoter promotes the development of TC, contributes to disease aggressiveness and treatment resistance, and thereby leads to poor patient outcomes. From a clinical point of view, the strong association between the TERT promoter mutation and disease malignancy and aggressiveness holds great promise for its value in TC diagnostics, risk stratification, prognostication, treatment decision, and follow-up design. In the present review article, we summarize the recent findings of studies of TERT promoter mutations in TC and underscore the implications of TERT hyperactivity driven by genetic events in the pathogenesis and management of TC. Finally, the targeting of TERT promoter mutations and the disruption of telomere maintenance are considered as potential therapeutic strategies against TC.
\end{abstract}

Keywords: Cancer biomarker; telomerase reverse transcriptase promoter mutations (TERT promoter mutations); telomerase; thyroid carcinoma (TC); thyroid nodule

Submitted May 07, 2020. Accepted for publication Aug 19, 2020.

doi: 10.21037/atm-20-5024

View this article at: http://dx.doi.org/10.21037/atm-20-5024

\section{Introduction}

Globally, thyroid carcinoma (TC) is the most common malignancy of the endocrine system, with an incidence that has at least doubled in the past two decades (1-3). In the vast majority of cases, TC (>95\%) originates from follicular thyroid cells (thyrocytes), while the remaining $<5 \%$ of cases are derived from calcitonin-producing parafollicular or C cells $(1,4,5)$. Thyrocyte-derived TCs are largely categorized into papillary (PTCs, up to $85 \%$ ), differentiated follicular (FTCs, 10-15\%), poorly differentiated (PDTCs)

\footnotetext{
^ ORCID: 0000-0003-3141-4524.
} 
(5-10\%), and anaplastic (ATCs) subtypes (2-3\%). Recent advances in high-throughput sequencing technologies have facilitated breakthroughs in the genomic, epigenetic, and transcriptomic landscapes of TC (6-9), and have thereby contributed to profound insight into its molecular pathogenesis. Different subtypes of TCs are characterized by different genetic alterations, resulting in a close genotype-phenotype correlation $(4,5,9)$. PTCs have a high frequency (up to $60 \%$ ) of activating $B R A F$ gene mutations (predominantly $\mathrm{BRAF}^{\mathrm{V} 600 \mathrm{E}}$ ), or RAS mutations or RET-PTC fusion, whereas most FTCs have PAX8-PPAR $\gamma$ fusion or RAS mutation, but rarely exhibit altered BRAF. Meanwhile, BRAF and RAS mutations both occur frequently in PDTCs and ATCs, and the inactivation of the tumor suppressor TP53 is common, especially in ATCs $(7,9,10)$. In addition, the aberration of other tumor suppressors including CDKN2A-RB1 and PTEN is seen in approximately onethird and one-tenth of ATCs, respectively $(7,9)$. The inactivation of the tumor suppressors is evidently a defining characteristic of undifferentiated PDTCs and ATCs, compared to differentiated FTCs or PTCs. Collectively, the identification of genomic and epigenetic alterations above, together with an increasing awareness of patient specific clinical features, has made precision medicine in TC care become a realized paradigm for disease diagnosis, risk stratification, prognostication, and treatment decision or targeted therapeutics.

As described above, in each of the four subtypes of TCs, most altered genes are involved in cell cycle regulation. The BRAF/RAS mutation or the fusion of RET-PTC/ PAX8-PPAR $\gamma$ triggers the MAPK signaling hyperactivation, while the inactivation of TP 53 or CDKN2A-RB 1 erases the checkpoint control for cell cycle progression, collectively promoting abnormal cellular proliferation $(5,9)$. However, none of these genetic alterations are sufficient for uncontrolled cell proliferation, which is a key hallmark of TC and other human malignancies (11); to achieve immortality, the intrinsic senescence barrier must be erased $(12,13)$.

The crucial roles telomeres and telomerase play in regulating the life span of human cells have been well established $(12,13)$. Telomeres are located at the end of linear chromosomes and are composed of 6-20 kb long TTAGGG repetitive sequences associated with telomere binding factors $(12,13)$. The TTAGGG repeat-protein structure of telomeres forms a protective cap by which chromosomes maintain genomic stability and integrity through inhibiting DNA damage response and illegitimate recombination of DNA (13). A key feature of telomeres is their progressive attrition with successive cell divisions caused by "the end-replication problem" and the lack of telomerase $(12,13)$. When telomeres become too short to exert their protective effect on chromosomes, dysfunctional telomeres mimic DNA damage to activate the DNA damage response cascade. Subsequently, tumor suppressive/ growth-inhibiting (checkpoint) genes are transcriptionally induced, and cellular senescence (mortality stage 1 or M1) occurs (13). In this process, TP53 and/or CDKN2A-RB1 checkpoint signaling is pivotal in triggering the senescence program $(12,13)$. Telomere shortening serves as a mitotic clock, recording times of cell replication and limiting their lifespan $(12,13)$. Thus, stabilizing telomere length and preventing senescence are vital steps toward infinite proliferation and malignant transformation, and telomerase activation has been shown as the most common strategy by which immortality in TC and other human malignancies is accomplished $(12,13)$. During the TC pathogenesis, telomerase may be activated via different mechanisms, among which the gain-of-function TERT promoter mutation is particularly important, due to its importance both biologically and clinically (12). The present review is thus focused on TERT promoter mutations in TC, thereby providing a fresh perspective on the molecular pathophysiology of this genetic event and its implications in precision medicine in TC care.

We present the following article in accordance with the Narrative Review reporting checklist (available at http:// dx.doi.org/10.21037/atm-20-5024).

\section{The general telomerase activation model in carcinogenesis and relation to TC}

Telomerase consists of multiple factors, but its essential components include a catalytic subunit telomerase reverse transcriptase (TERT) and an internal telomerase RNA template (TERC). TERC is ubiquitously present in human cells, whereas the TERT gene is tightly repressed in most human somatic cells, including thyrocytes, resulting in telomerase silencing (12-14). In carcinogenesis, telomerase is activated via the induction of TERT expression (14). The underlying mechanisms of TERT transcription and telomerase activation have been explored in many studies.

In the overwhelming majority of human malignancies, telomerase is activated via TERT induction, but telomeres in malignant cells are in general shorter than those in their normal counterparts $(12,13)$. This apparently paradoxical 
scenario can likely be attributed to the late activation of telomerase in tumorigenesis. In in vitro studies, telomerasedeficient, proliferative cells possessing functional TP53 and CDKN2A-RB1 signals were shown to undergo telomere shortening and eventually enter into a senescent M1 stage. However, when these pathways are inactivated by carcinogens, cells are able to bypass M1 and continue to proliferate, even if telomeres become dysfunctional, until an mortality stage 2 (M2) crisis occurs $(12,13)$. Upon reaching an M2 crisis stage, cells exhibit drastic genomic catastrophes, including breakage-fusion-bridge cycles, endto-end fusion, chromosome rearrangement, and many other aberrations. As a result of telomerase activation through TERT induction, the vast majority of these cells undergo apoptosis, with only a few surviving (13). Activated telomerase maintains telomeres at a shorter length balance and attenuates chromosomal instability to a level that cells are able to tolerate; however, telomerase activity in these cells is usually insufficient for all telomeres to increase to the pre-senescent length (13). Nevertheless, this telomerase activity facilitates the infinite proliferation of cells. Analyses of human tissues and primary cells during a stepwise carcinogenesis process have supported this in vitro M1 senescence and M2 crisis cell model $(15,16)$.

Shorter telomeres coupled with telomerase activation are similarly observed in TC (17), and the abovedescribed cancer-related telomerase activation model may to a large extent also fit the TC pathogenesis. Normally, thyrocytes, with an estimated turnover of five times during a human lifetime, do not divide actively in vivo (18); however, oncogenic events frequently induce the activating mutation of the $B R A F$ or $R A S$ gene (5), thereby generating a hyperproliferative signal and forcing cells to replicate far beyond the physiological lifespan of thyrocytes. In de novo ATC, in which TP53 and/or CDKN2A-RB1 tumor suppressors are inactivated and genetic aberrations are widespread, it is conceivable that precursor cells bypass senescence and undergo M2 crisis before their transformation into ATC. However, this scenario may be extremely different from differentiated FTC and PTC, in which the TP53 and CDKN2ARB 1 pathways are generally maintained (5). The introduction of the oncogenic BRAF or RAS into human normal thyrocytes or other cells is known, through the activation of TP53 and/or CDKN2A checkpoints, to result in oncogene-induced senescence (OIS) (19). OIS is an intrinsic mechanism for tumor suppression and must be erased during the development of PTC and
FTC. Zou et al. recently showed that thyroid-stimulating hormone (TSH) played a pivotal role in overcoming $\mathrm{BRAF}^{\mathrm{V} 600 \mathrm{E}}$-induced OIS in the pathogenesis of PTC by inhibiting TP53 (20). In a TC mouse model, BRAF ${ }^{\mathrm{V} 600 \mathrm{E}}$ alone was insufficient for inducing PTC due to the onset of OIS, and its promotion of PTC formation and progression depended on its interaction with TSH $(20,21)$. Serum TSH levels have consistently been observed to be significantly higher in TC patients than in healthy individuals (22). Thus, TSH-mediated TP53 inhibition likely enables the continuous proliferation of thyrocytes driven by oncogenic signals until an M2 crisis is reached, through which telomerase is activated.

Questions still surround how TERT expression is induced during an M2 crisis or carcinogenesis $(12,13)$. Based on the above-described model, TERT induction/ telomerase activation is the integrated part of the whole oncogenic program. Moreover, as the transcriptional silence of the TERT gene is a fundamental mechanism in the suppression of telomerase activity in normal cells, de-repressing the TERT gene is certainly a vital step in the activation of telomerase in transformed cells (12). Thus, the gain of oncogenic signals or loss of tumor suppression function contributes to the activation of TERT transcription. For instance, MYC and $\beta$-Catenin, which are frequently dysregulated in TC $(5,23)$, are two well characterized oncogenic factors that directly transactivate the TERT gene (24-27). Meanwhile, TP53 inhibits TERT expression by abolishing transcription factor Sp1 binding to the TERT promoter (28). Recently, the comprehensive characterization of human cancer genomics has unearthed novel mechanisms by which TERT transcription and telomerase are activated, including recurrent promoter mutations, focal rearrangements, and onco-viral DNA insertion within the TERT locus (12). In each of these genetic events, TERT promoter mutations are paramount in the pathogenesis of TC $(29,30)$.

\section{The TERT promoter mutation for telomerase activation in TC}

The recurrent TERT promoter mutations, C228T and C250T predominantly occur at two sites with a cytidineto-thymidine $(\mathrm{C}>\mathrm{T})$ dipyrimidine transition in the proximal region of the TERT promoter $(-124$ and -146 bp from ATG, respectively) $(31,32)$ (Figure 1A). These mutations were first reported in TC in 2013 by Liu et al., who observed that the mutation frequency was $12 \%$, 
14\%, 38\%, and 46\% in PTCs, FTCs, PDTC,s and ATCs, respectively (34). Largely consistent with their results, we showed that the mutations occurred in $17-22 \%$ of FTCs, 25\% PTCs, and 50\% ATCs (17). Landa et al. observed similar mutation frequencies (35). To date, almost 200 related research articles have been published, involving more than 15,000 patients from around the world who were analyzed for their TERT promoter mutation statuses and clinical significance in TC. Based on these clinical observations, the following conclusions can be drawn: (I) disease aggressiveness and mutation rates are intimately correlated. The most aggressive TCs, including ATCs and PDTCs, exhibit the highest frequency of TERT promoter mutations; (II) mutations in young patients ( $<45$ yrs old) are rare, and especially in pediatric TC cases; (III) C228T is the predominant mutation in $\mathrm{TC}$, and the presence of $\mathrm{C} 228 \mathrm{~T}$ and C250T is mutually exclusive. In rare cases, C228T and C250T may occur in different subclones or cell populations within the same tumor (36); (IV) ethnic and geographic differences in TERT promoter mutations exist in TC. In a cohort of Greek PTC patients, Argyropoulou et al. reported the mutation rate as 3.4\% (2/59) (37), and two Chinese groups recorded mutation rates of $2.0 \%(7 / 355)$ and $3.5 \%(12 / 342)$ in cohorts of PTC patients from the Han population $(8,38)$; these rates are much lower than those (from $12 \%$ to $25 \%$ ) reported in other studies $(7,17,34$ ). A recent meta-analysis including 11,382 patients worldwide found the average TERT promoter mutation rates for PTC and FTC patients to be $10.6 \%$ and $15.1 \%$, respectively (39).

Primary tumors harboring the TERT promoter mutations display high levels of TERT and telomerase activity $(17,33,40)$. Based on the analysis of pooled cancers from the TCGA dataset, TERT expression and the telomerase score were significantly higher in tumors carrying the mutant TERT promoter than in their wtpromoter-bearing counterparts (41). In PTC, we found that all of the tumors carrying the mutant TERT promoter expressed TERT mRNA, while TERT expression was detected in only $38 \%$ of tumors with a wt promoter (33). Mechanistically, both C228T and C250T mutations result in the generation of a de novo binding motif for the ETS family of transcription factors through which TERT expression is transcriptionally induced (31) (Figure 1A). A number of ETS transcription factors have been shown to activate TERT transcription in tumor cells harboring mutant TERT promoters (42), although ETS family members GA-binding proteins A and B (GABPA and GABPB) were identified as master driving factors of mutant TERT promoter activity (43-45) (Figure 1A). Consistently, the inhibition of GABPA or GABPB1 sees the downregulation of TERT expression (33,43,45-47).

GABPA is a special member of the ETS family, because it contains only a DNA binding domain and is incapable of activating its target genes alone (48). To accomplish this task, GABPA works with its partners GABPB1 or GABPB2 to form the complex that harbors a transactivation domain $(47,48)$. The GABPA and GABPB complex in general binds to its ETS motifs as a heterotetramer [(GABPA/GABPB $)_{2}$ ] $(47,48)$ (Figure 1A). Interestingly, Liu et al. (45) showed that the transcription factor FOS was phosphorylated and activated by the hyperactive MAPK signalling mediated by $\mathrm{BRAF}^{\mathrm{V} 600 \mathrm{E}}$; in turn, FOS facilitated the transcription of its target gene GABPB1, thereby enhancing TERT expression in mutant TERT promoter-carrying TC cells. These findings provide a functional link between BRAF ${ }^{\mathrm{V} 600 \mathrm{E}}$ and mutant TERT promoter, and explain why these two genetic events frequently co-exist in PTC. On the other hand, mutant TERT promoter-harboring glioblastoma cells depleted of GABPB1 were found to have reduced TERT expression, progressively shortened telomere or telomere dysfunction, defective proliferation/survival, and eventual loss of tumorigenic potential (49). With these results considered, GABPA/B1 has been suggested as a therapeutic target for mutant TERT promoter-carrying malignancies (49).

However, an unexpected, more complicated relationship between GABPA and TERT has been revealed by the recent results of studies on TC, urotheliumderived bladder cancer (BC), and hepatocellular carcinoma (HCC) $(33,46,47,50)$. First, in both wt and mutant TERT promoter-bearing TC cells, TERT expression was inhibited by GABPA depletion (33), which strongly suggests that GABPA modulates the TERT transcription independently of TERT promoter mutations in these cells. Furthermore, in primary PTC tumors, GABPA expression was inversely correlated with the presence of TERT promoter mutations or TERT mRNA levels (33). Second, GABPA inhibition robustly promoted the invasion, stemness, and/or drug resistance of TC, BC and HCC cells via different mechanisms $(33,47)$ (Figure 1B). Moreover, a close association between lower GABPA expression and metastasis and poor outcomes in TC patients has been established (33) (Figure 1C). Together, these observations indicate that the effect of GABPA depends on context and, despite its stimulatory effect on TERT expression, it may function as a tumor suppressor in TC, 
A

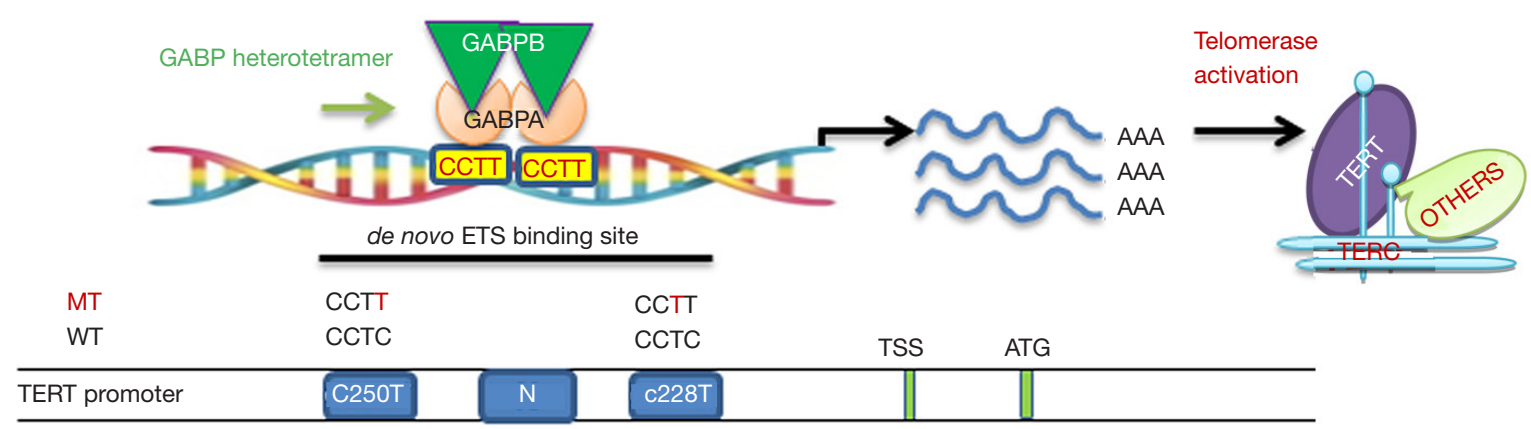

B
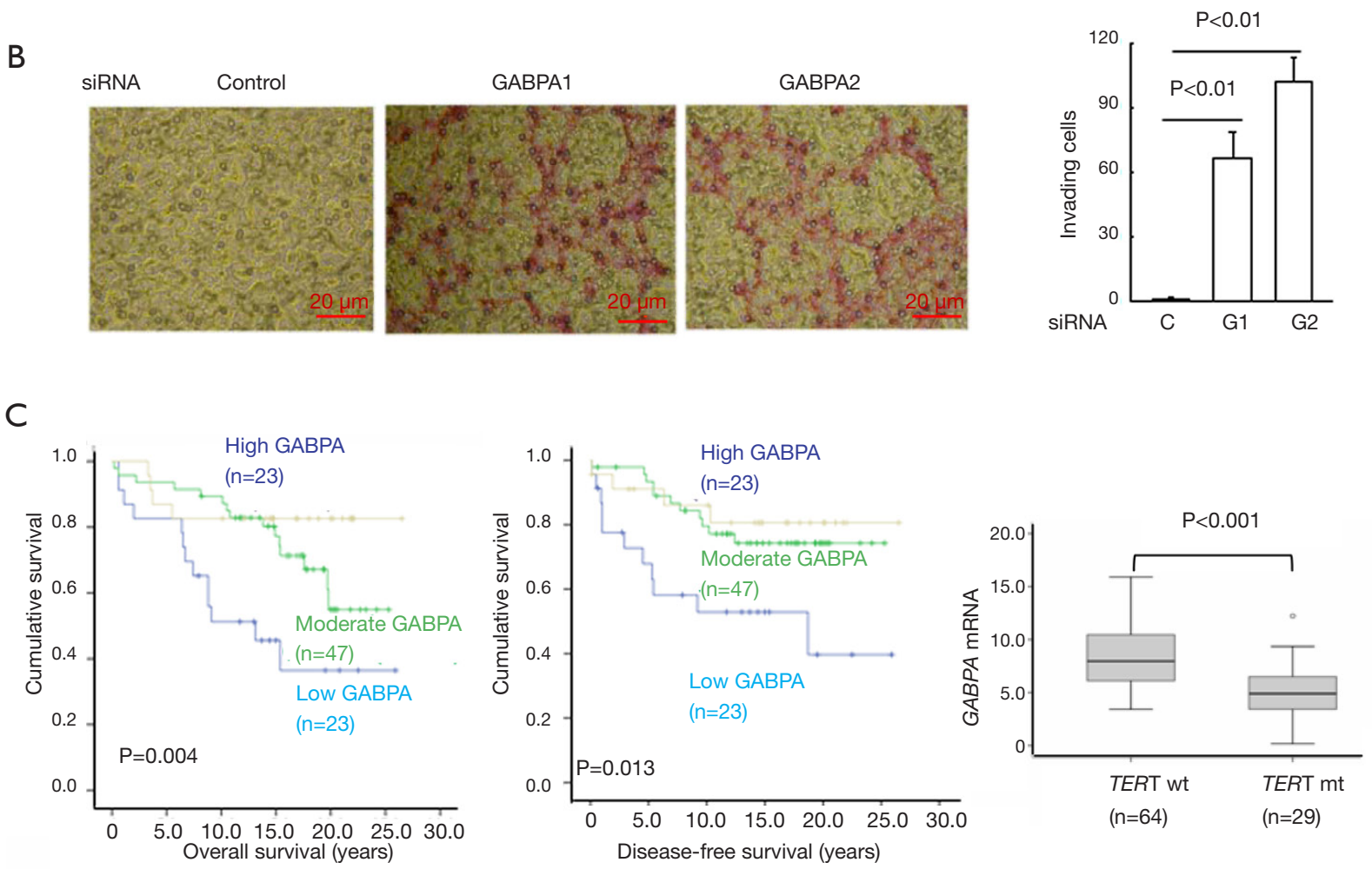

Figure 1 TERT promoter mutations/telomerase activation, GABPA/B transcription factors and their relation to TC. (A) TERT promoter mutations/telomerase activation and their transcription activator GABPA-B complex. C>T mutation occurs at one of two positions of the TERT proximal promoter (-124 and -146 to ATG for C228T and C250T, respectively) in TC and other malignant cells. The mutation creates a de novo ETS binding motif, and the ETS family members GABPA and GABPB1 or GABPB2 form a complex that binds to the de novo ETS site, and thereby activates the transcription of the TERT gene. The induced TERT gene together with ubiquitously expressed TERC and other components form an active telomerase complex for telomere lengthening. Of note, the presence of two ETS motifs close to each other is preferably recognized and bound by the GABP complex, and two complexes further form a heterotetramer (GABPAGABPB)2 for stronger transcriptional activity. N: Native ETS site in the TERT promoter. (B) GABPA depletion robustly promotes the invasiveness of ATC-derived cells harbouring C228T. ATC-derived cells were transfected with scramble control and two different GABPAspecific siRNAs, respectively; these cells were then analyzed for their invasion capacity. (C) The association between GABPA expression and survival and TERT promoter mutations in PTC patients. The analysis included 93 PTC patients and higher GABPA expression predicted longer overall and disease-free survival (left and middle panels). The PTC tumors bearing the mutated TERT promoter expressed significantly lower levels of GABPA (right). (B,C) panels was reproduced from (33) with permission from Springer Nature. TERT, telomerase reverse transcriptase; TC, thyroid carcinoma; ATC, anaplastic thyroid carcinoma; PTC, papillary thyroid carcinoma. 
$\mathrm{BC}$, and HCC. In that case, if GABPA serves as a target by which to inhibit telomerase in cancer therapy, tumor dissemination may occur far before cancer cells undergo senescence/apoptosis mediated by telomerase inhibition and telomere dysfunction. Thus, it is currently premature to propose GABP factors as a target for telomerase-based cancer therapies.

\section{The TERT promoter mutation as an early genetic event in the TC pathogenesis}

According to the telomerase activation model described above, the induction of TERT expression and telomerase activity in carcinogenesis occurs at a later stage (13); however, we previously identified C228T mutation in premalignant thyroid lesions (40), especially in atypical follicular thyroid adenoma (AFTA) (51) or follicular tumors of uncertain malignant potential (FTUMP) (52), and the mutation frequency in AFTA/ FT-UMP is equal to that seen in FTC $(17 \%)(40,53)$. Moreover, all C228T-bearing AFTA/FT-UMP tumors display TERT mRNA and telomerase activity, which demonstrates that the mutation is functional (40). Despite the presence of active telomerase, however, these tumors harbor much shorter bulk telomeres than their mutation-lacking counterparts do, which indicates that the TERT mutation is insufficient for supporting telomere stabilization and full malignant transformation in these AFTAs/FT-UMPs (40). However, it is also possible that shorter or dysfunctional telomeres preferably drive TERT promoter mutations. Nevertheless, our findings clearly show that the TERT promoter mutation is an early genetic event in the initial stage of TC development. Consistent with our report, Hosen et al. (54) recently observed that the TERT promoter mutation could be already be detected in urine 10 years before clinical diagnosis of $\mathrm{BC}$. Also, the mutation was observed in premalignant lesions of other human malignancies (30). Collectively, these results suggest that the TERT promoter serving as an oncogenic target for mutations may be a general phenomenon during the early stages of carcinogenesis.

Similarly, a recent comprehensive analysis of the pathogenesis of melanoma showed that the TERT promoter mutation occurs early but is insufficient for maintaining telomere length during the evolution from benign nevus to malignant melanoma (16). In the early phase, the mutationmediated telomerase expression is only able to lengthen the shortest telomeres and thereby to extend the lifespan of precursor cells. Continuous proliferation drives cells to enter an M2 crisis, when genomic catastrophes are induced and telomerase is subsequently further upregulated to the level at which an immortal phenotype is acquired and malignant conversion is fully accomplished. Accordingly, the authors suggest a two-step model for telomerase activation in the development of melanoma.

The underlying mechanism of TERT induction/ telomerase activation in TC, however, is more complicated. The two-step model may be applicable to PDTC and ATC, but PTC and FTC involve different processes. The results from our cohort of PTC patients and a TCGA dataset showed that most wt TERT promoter-carrying PTC tumors (close to 2/3) expressed undetectable or negligible levels of TERT. These PTCs may only avoid OIS resulting from TSH-mediated TP53 inhibition, but without undergoing M2. This lack of TERT expression enables the disease indolence and rare recurrence after treatment. In contrast, all mutant TERT promoter-carrying PTCs express TERT, and are associated with widespread genomic aberrations $(41,55,56)$. Moreover, a fraction of these PTCs transform into dedifferentiated, more aggressive ATCs expressing more abundant amounts of TERT and telomerase activity (10). Collectively, at least a subset of PTCs might have undergone M2 crisis to further robustly upregulate TERT expression. On the other hand, one-third of PTCs lacking TERT promoter mutations express TERT, and it is likely that other TERT regulators are activated either before or after M2 crisis. Taking all these scenarios into consideration, we suggest a TERT/telomerase activation model for TC in Figure 2.

\section{Telomere dysfunction as a potential driver for the TERT promoter mutation in TC}

The exact mechanism by which TERT promoter mutations occur in TC and other malignancies is still unclear. Several lines of evidence indicate shorter or dysfunctional telomeres to be a key driving force: (I) The mutation is predominantly seen in senior-age TC patients and is rarely observed in pediatric or young TC patients (17). Because telomeres shorten progressively with increased age, thyrocytes in younger individuals have longer telomeres compared with those in older people, and they are under less pressure from telomere erosion mediated by oncogenic events (17). (II) In both AFTA and TC, telomeres are significantly shorter in tumors carrying TERT promoter mutations than those without mutations $(17,40)$. Moreover, the presence 


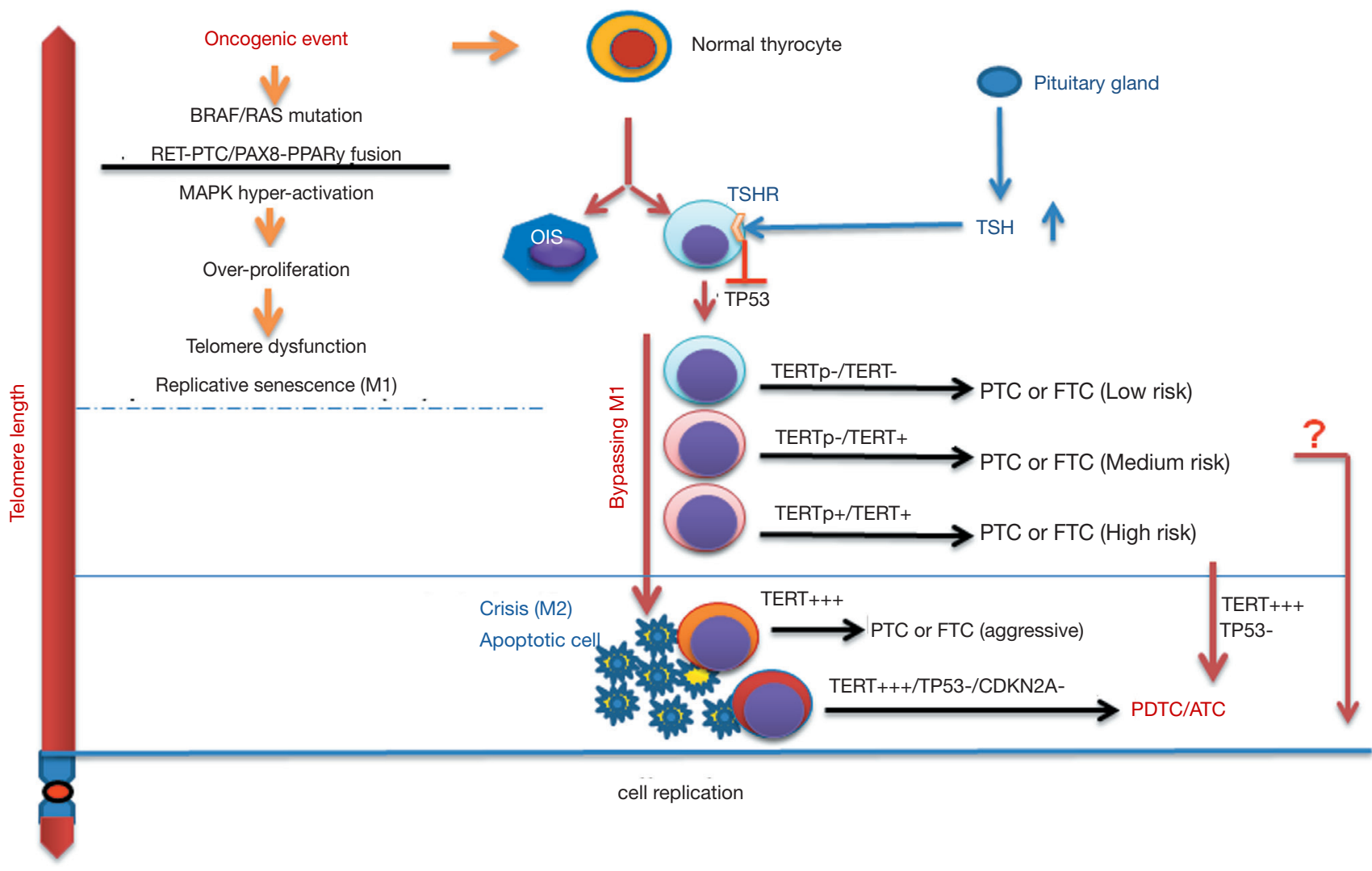

Figure 2 The model for TERT promoter mutations, TERT expression and telomerase activation during the pathogenesis of TC. Oncogenic events target BRAF/RAS for their mutations or RET-PTC/PAX8-PPAR $\gamma$ for their fusion in thyrocytes. These mutations or fusions result in the hyperactivation of the MAPK pathway and drive the thyrocytes to over-proliferate, which in turn leads to premature cell senescence so-called OIS. However, the pituitary gland may secrete increased amounts of TSH, through which TP53 function is inhibited. When this happens, cells bypass senescence or M1 phase and continue to proliferate, causing telomere dysfunction. Shortened telomeres are a key driving-force in triggering TERT promoter mutations and TERT expression. This group of patients with PTC/FTC may undergo M2 crisis and advance to PDTC or ATC or more aggressive PTC/FTC. De novo PDTCs and ATCs are most likely derived from their precursor cells that have undergone M2, and these cells acquire widespread TP53 inactivation and robust upregulation of TERT expression and telomerase activity. However, the majority of PTCs/FTCs lack TERT promoter mutations and many of them do not express TERT, but they have relatively longer telomeres in the tumors, and the disease is indolent. A minority of PTCs/FTCs express TERT mRNA, and a fraction of them undergo M2 crisis and progress. Conceivably, TERT/telomerase-deficient PTCs/FTCs are transformed without experiencing M2 crisis and thus lack significant chromosome aberrations. TERT, telomerase reverse transcriptase; TC, thyroid carcinoma; OIS, oncogene-induced senescence; TSH, thyroid-stimulating hormone; PTC, papillary thyroid carcinoma; FTC, follicular thyroid carcinoma; PDTC, poorly differentiated thyroid carcinoma; ATC, anaplastic thyroid carcinoma.

of TERT promoter and BRAF/RAS mutations is highly concordant, which suggests that the hyperactivation of MAPK signaling forces thyrocytes to over-proliferate, driving massive telomere erosion $(34,35,45,57,58)$. Indeed, the TERT promoter mutation in PTC is intimately correlated with chromosomal aberrations and mutational burdens (55). (III) The germline variants at the TERT locus significantly affect the occurrence of TERT promoter mutations in cancer patients, and the TERT rs2736100-CC cancer risk genotype is associated with longer telomeres and is seen less frequently in patients with a mutant TERT promoter (59). This variant is known to upregulate TERT expression, thereby counteracting the shortening of telomeres (60). (IV) Finally, in non-cancerous individuals 
who suffer from germline defects in TERT or other telomerase components, extremely short telomeres trigger the spontaneous acquisition of TERT promoter mutations in their lymphocytes (61). Importantly, C228T or C250T mutation can normalize telomerase activity and increase the proliferation potential of those cells (61). In patients harboring a heterozygous mutation in the TERT coding region, a clear selection bias exists toward cells in which the activating promoter mutation drives the expression of wt, but not the mutant TERT allele (61).

The TERT locus is located at chromosome $5 \mathrm{p}$, and is only a megabase away from the $5 \mathrm{p}$ end. A recent report showed that TERT expression is regulated by telomere length, a telomere position effect-like mechanism, or telomere position effect-over long distances (62). In cells with long telomeres, a telomere-loop structure forms in the region near the TERT locus, leading to a repressive TERT chromatin at the promoter; however, when $5 \mathrm{p}$ telomere becomes critically short, this loop is disrupted, thereby inducing a loose TERT chromatin that is easily accessible for oncogenic elements or mutagens. Such a scenario leads to the onset of promoter mutations. This hypothesis calls for validation in experiments.

\section{The TERT promoter mutation: Beyond telomere- lengthening function in TC}

TERT promoter mutations are significantly associated with transcriptomic alterations in PTCs (55). The expression of genes that control DNA damage response and cell cycle progression is highly enriched, while transporter or metabolic activities are downregulated in mutant TERT promoter-bearing tumors (55). Luo et al. achieved similar results in their study, in which PTC patients were categorized into TERT ${ }^{\text {High }}$ and TERT ${ }^{\text {Low }}$ groups based on their TERT mRNA levels (56). The two groups were shown to exhibit substantial differences in their DNA methylation profiles (56). Thus, it is conceivable that altered transcriptome can be attributed to aberrant DNA methylation (63). In line with these findings, we recently observed that TERT interacted with the transcription factor Sp1 to promote transcription of the DNA methyltransferase DNMT3 gene, thereby contributing to the silencing of the tumor suppressors PTEN and RASSF1A via the induction of their promoter hypermethylation (64). TERT has been shown to serve as a transcription co-factor, which interacts with a panel of transcription factors to facilitate the target transcription essential for oncogenesis (64-66). By doing so,
TERT not only confers cancer cells an immortal phenotype, but also empowers them with stemness, invasive phenotype, and drug resistance or anti-apoptotic insults (12,64-69). Therefore, TERT exerts its oncogenic functions in both telomere lengthening-dependent and independent manners, thereby promoting the development and progression of TC.

\section{The TERT promoter mutation as a diagnostic marker for TC}

Distinguishing between benign and cancerous thyroid nodules is crucial to avoiding unnecessary treatment for patients with benign conditions and to providing appropriate interventions for those with TC. Cytological examination of collected cells through fine needle aspiration biopsy (FNAB) facilitates an accurate diagnosis in most patients; however, as many as $30 \%$ of cases may prove inconclusive, with indeterminate morphology, and many of these patients thus undergo needless diagnostic lobectomy. Because TERT promoter mutations are present specifically in TC and AFTA, but are rarely encountered in benign tumors and are absent in normal thyroid tissues, they are believed to be specific biomarkers in TC diagnosis. Using Sanger sequencing, Liu et al. analyzed TERT promoter mutations in DNA specimens from FNAB of 179 benign and 129 malignant thyroid nodules and discovered that 9/129 TC nodules and none of the benign nodules were positive for the mutation, representing $100 \%$ diagnostic specificity (70). Cancer-specific TERT promoter mutations were similarly observed in other FNAB studies (71-73). Using core needle biopsy (CNB), Crescenzi et al. reexamined thyroid nodules with inclusive reports caused by poor FNAB sampling and identified the presence of C228T mutations in $17 \%$ of locally advanced PTCs (74). While CNB is more invasive than FNAB, it allows more accurate assessment of TERT promoter mutations and pathological variables for nodule diagnostics, intervention, surgical options, and follow-up design. Therefore, if FNAB is inconclusive, CNB is strongly recommended before diagnostic surgery is opted for.

For most patients with benign thyroid nodules, diagnostic surgery is unnecessary, although post-operative screening of the TERT promoter mutation in resected specimens is beneficial. The presence of the mutation helps to identify those with malignant/aggressive potential, even in the absence of pathological and morphological evidence of malignant behavior. Hysek et al. (53) analyzed TERT 
promoter mutations in AFTA/FT-UMP tumors from 51 patients, finding that 7 had C228T and 1 had C250T. Fullblown malignant FTC was eventually developed by 3 out of 8 patients, whereas none of the patients with a wt promoter experienced disease progression. Notably, the pathological examination of diagnostic hemithyroidectomy in one patient even showed a feature typical of FTA (53). Thus, TERT promoter mutation analyses are extremely useful for risk stratification in patients with FTA/FT-UMP.

Since the TERT promoter mutation assay is not readily available in every hospital, ultrasound examinations, as a clinical routine practice, and clinicopathological features have been combined to predict the presence of TERT promoter mutations in PTC. Shi et al. showed that in patients aged $>50$ years old, multifocality, a taller-thanwide shape, microlobulated margin, and capsule contact or involvement were strong indicators that a tumor harbored TERT promoter mutations (75). In other clinical observations, hypoechogenicity, non-parallel orientation, spiculated/microlobulated margin, and micro-calcifications have been identified as indicators of the existence of TERT promoter mutations (76,77). However, these results are largely preliminary, which need further validation through recruiting and analyzing larger cohorts of TC patients. More recently, deep learning-based classification of ultrasound images has been applied for thyroid nodule diagnosis and highly accurate recognitions of the changes documented above (78). It will be interesting to assess if the application of this approach will be able to predict PTCs with TERT promoter mutations more precisely in future.

There are a number of issues to address at this point: (I) the frequency of TERT promoter mutation in PTC and FTC is reported to range from $2.4 \%$ to $25 \%$, which constitutes a low sensitivity for this detection biomarker in TC (39). Therefore, the exclusion of malignancy should not be based on undetectable TERT promoter mutations only. The combined assessment of the mutant TERT promoter with other genetic biomarkers (e.g., BRAF ${ }^{\mathrm{V} 600 \mathrm{E}}$ ) is expected to greatly improve the accuracy of thyroid nodules diagnosis (70). (II) Sanger sequencing, which was utilized in the studies above, is the gold standard for the identification of mutant targets, but its threshold sensitivity is rather low: at least $10 \%$ of mutant TERT promotercontaining tumor DNA is needed for a level detectable by Sanger sequencing to be reached (79). Moreover, tumor heterogeneity is widespread, which may further compromise the accuracy of the assay. Analysis approaches with high sensitivity need to be developed to overcome such technical problems. CastPCR and ddPCR have been shown to robustly raise detection sensitivity without compromising specificity $(79,80)$, and these approaches have proven useful for assessing TERT promoter mutations in FNAB or CNB specimens. Also, next-generation sequencing (NGS) was recently employed to analyze a large panel of driver mutation genes, including ThyroSeq v2 with a mutant TERT promoter, in TC. NGS allows for the highly accurate diagnosis of thyroid nodules, although it is not time or cost friendly (81). Similar results have also been demonstrated by independent groups using NGS or different mutation signatures $(82,83)$. In short, the development of highly sensitive, specific and simple assays suitable for routine practice (like ultrasound) is urgently needed from a clinical point of view.

\section{The TERT promoter mutation as a prognostic marker for TC}

Clinical studies of TC patients have revealed that the presence of TERT promoter mutations is highly correlated with advanced or progressive disease, including advanced stages, extrathyroidal extension, vascular invasion, lymph node metastases, distant metastases, and recurrence $(10,17,29,30,34,39,67)$. Given these findings, along with the multi-oncogenic activities of TERT/telomerase, TERT promoter mutations are expected to significantly affect the outcomes or mortality of TC patients. Indeed, direct evaluation of the TERT promoter mutation and patient outcomes has consistently shown that the mutation serves as an independent factor in predicting shorter disease-free survival (DFS) in TC $(57,58,67,70,84-94)$ (Figure 3).

Most PTC tumors harbor a BRAF ${ }^{\mathrm{V} 600 \mathrm{E}}$ mutation, and its value for predicting prognosis has proved controversial due to discrepant results in different clinical observations $(84,88,93,95,96)$. It is now clear that this genetic aberration alone may not significantly affect patient outcome; however, its co-existence with the TERT promoter mutation leads to the formation of the most aggressive PTC tumors and even promotes the evolution of PTC to deadly ATC. Liu's and Xing's groups recently showed that this combination could be applied to robustly stratify the PTC patients with the highest mortality risk and predict the patient with the shortest DFS $(57,58,70)$. Their findings have been replicated in other independent observations $(85,87,91,94,97-99)$. In addition, a similar effect on PTC/FTC mortality was observed in the co-existing mutant RAS and TERT promoter mutations $(87,90,94,98)$. Molecularly, mutant 

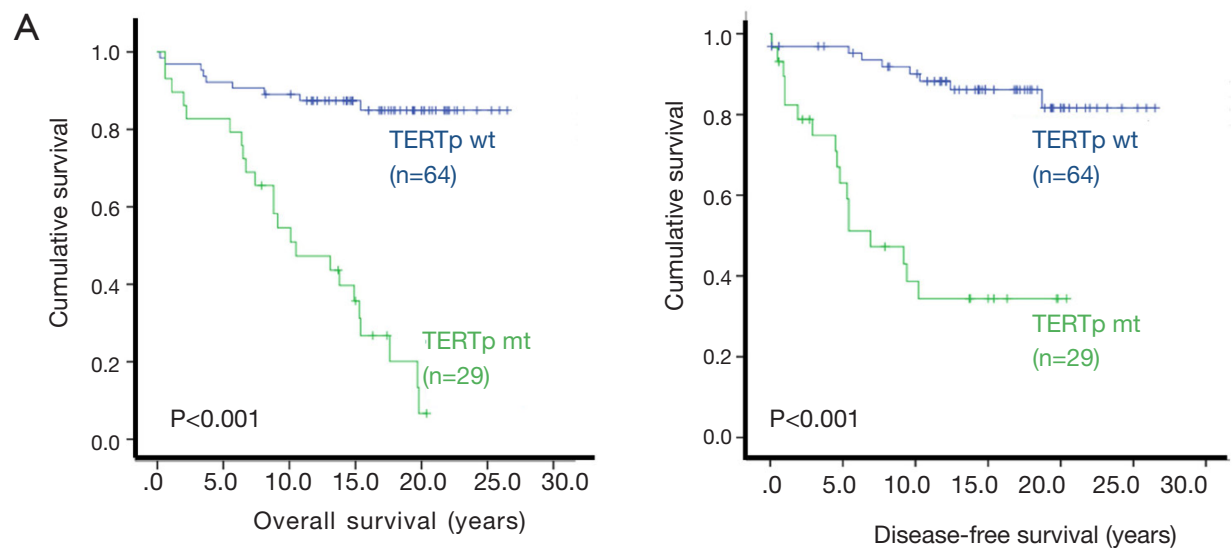

B
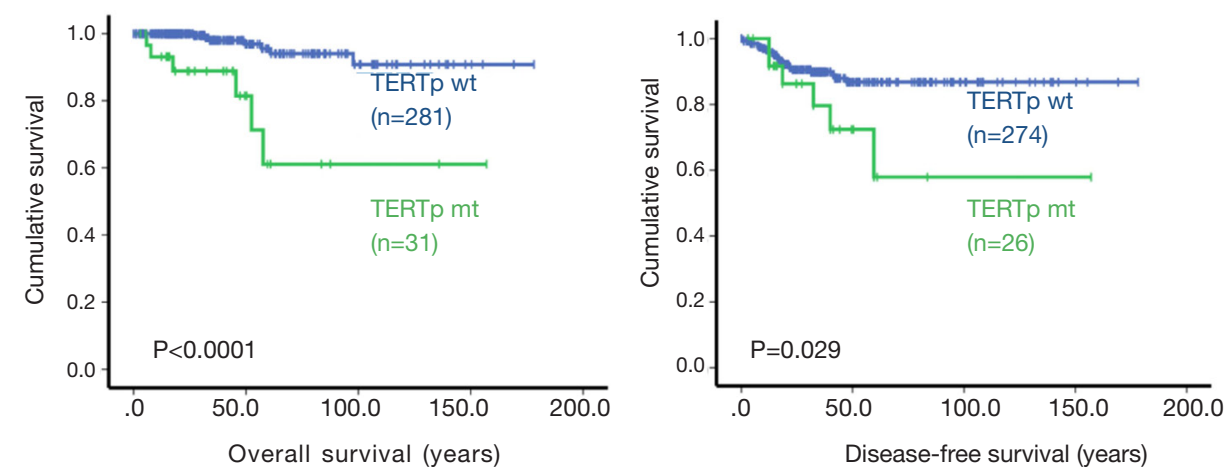

Figure 3 The mutated TERT promoter as a prognostic marker in patients with PTC. (A) The overall and disease-free survival of 93 PTC patients was compared between their tumors with wild type (wt) and mutated (mt) TERT promoter (TERTp). The presence of mt TERTp predicts significantly shorter overall and disease-free survival. The data were reproduced from (33) with permission from Springer Nature. (B) The survival analysis was performed on the TCGA cohort of PTC patients. The patient data was downloaded from ciBioPortal for Cancer Genomics (www.ciBioPortal.org) in Jan. 2019. The numbers of patients available for overall and disease-free survival analyses were 312 and 300 , respectively.

BRAF/RAS-mediated hyperactivated MAPK signaling and over-proliferation drives genomic instability, while TERT promoter mutations and expression contribute to the acquisition of multi cancer-hallmarks in both telomere lengthening-dependent and independent manners (12). Thus, BRAF ${ }^{\mathrm{V} 600 \mathrm{E}}$ and the mutant TERT promoter synergize to amplify oncogenic signaling, accelerating TC development and progression (9).

\section{TERT promoter mutations and TC therapy}

The detrimental effect of the TERT promoter mutation on the outcomes of TC patients, as described above, may contribute to poor patient response to treatment. Loss of radioactive iodine (RAI) avidity in primary or recurrent PTC has been shown to be a major cause of treatment failure and unfavourable outcome. Yang et al. observed a significant correlation between the presence of TERT promoter mutations and decreased ${ }^{131} \mathrm{I}$ uptake and the RAI resistance categories of absent or weaker ${ }^{131} \mathrm{I}$ uptake (100). Other studies have demonstrated that the coexistence of $\mathrm{BRAF}^{\mathrm{V} 600 \mathrm{E}}$ and TERT promoter mutations is strongly associated with loss of RAI avidity and impairment of the iodide-metabolizing machinery in recurrent PTC $(94,101)$. Taken together, the presence of TERT promoter mutations may be used as an early predictor of radioiodine-refractory cases.

However, Tan et al. showed that the presence of the 
TERT promoter mutation sensitized $\mathrm{BRAF}^{\mathrm{V} 600 \mathrm{E}}$-bearing TC and other cancer cells to undergo apoptosis mediated by the combined treatment of $\mathrm{BRAF}^{\mathrm{V} 600 \mathrm{E}}$ and MEK inhibitors (102). The inhibitors induced robust apoptotic death of those cells carrying both TERT promoter and BRAF $^{\mathrm{V} 600 \mathrm{E}}$ mutations, while had negligible impacts on survival of cells bearing only BRAF ${ }^{\mathrm{V} 600 \mathrm{E}}$. Similar effects were also observed in xenograft mouse cancer models. As TERT is known to protect cancer cells from apoptosis induced by various insults (12), the finding above is unexpected. It is currently unclear how this happens; nevertheless, if validated, this observation should be very helpful for personalized TC treatment.

On the other hand, given the critical role of TERT promoter mutations in the pathogenesis of aggressive TC tumors, it is reasonable that this mutation or TERT/ telomerase is directly targeted in TC therapy. When a CRISPR method was used to correct the C228T mutation to wt C228C in C228T-bearing glioblastoma cells, these cells underwent reduced TERT transcription and TERT expression, and eventual senescence (103). To date, there has been no efficient treatment developed for ATC; however, the direct correction of the mutant promoter may hold promise for the future intervention in ATC. In addition, BRAF and/or MEK inhibitors have been applied to treat RAI-refractory and recurrent TCs in a phase III clinical trial, and improved survival has been documented, but such benefits are greatly compromised by the side effects of the drugs (104). Because the co-presence of $\mathrm{BRAF}^{\mathrm{V} 600 \mathrm{E}}$ or mutant RAS and TERT promoter mutations contributes to the aggressiveness of PTCs/FTCs, the combination of both BRAF and telomerase inhibitors might be more efficient, with less toxicity. In vitro experiments have shown that the inhibition of TERT by RNA interference (RNAi) or small molecule BIBR1532 leads to reduced invasion/ migration and increased senescence of TC cells $(67,105)$. In a xenograft mouse model, the administration of TERTspecific RNAi in nanoparticles efficiently blocked ATC cell growth (105); however, the in vivo inhibitory activity of BIBR1532 on TERT is extremely limited (13). These preclinical studies have demonstrated the feasibility and good efficacy of the targeting of TERT promoters and TERT mRNA/protein as a potential therapeutic strategy against TC, although there is a long way to go before it can be clinically applied.

Another telomerase inhibitor, Imetelstat (or GRN163L), a synthetic 5' palmitoylated 13-mer thiophosphoramidate oligonucleotide that disrupts the template function of telomerase RNA (13), has been shown to efficiently inhibit telomerase activity in various types of malignant cells and is the only telomerase inhibitor included in clinical trials (13). GRN163L treatment in myeloproliferative neoplasia has proven highly effective, with most patients undergoing rapid and durable hematologic and molecular remission following drug administration (106-108). It is especially important to note that the telomeres of mutant TERT promoter-carrying TC tumors are very short, which makes them more vulnerable to disruption of telomere length maintenance by GRN163L $(13,17)$. Therefore, the application of GRN163L for TC is likely to be an effective therapeutic approach. Exploring whether BRAF inhibitors and GRN163L are an efficient combination for PTC therapy will also be of interest.

\section{Conclusions}

TERT promoter mutations have been extensively investigated in TC. The results of these studies have not only led to a profound mechanistic understanding of the pathogenesis of TC, but have also provided useful tools for patient management. The TERT promoter mutation has been confirmed as an excellent biomarker for diagnosis, risk stratification, prognostication, treatment decision-making, and follow-up design in TC; however, studies on TERT promoter mutation- or telomerase-based TC therapy have fallen behind. Given their critical role in aggressive TC tumors, the targeting of TERT promoter mutation and telomere maintenance are expected to hold great promise for TC intervention and are worthy of further investigative efforts.

\section{Acknowledgments}

Funding: This work was supported by grants from China Postdoctoral Science Foundation Grant (2019M652404 and 2020T130372), the Swedish Cancer Society, Swedish Research Council, the Cancer Society in Stockholm, Karolinska Institutet Foundation, Swedish Foundation for International Cooperation in Research and Higher Education (STINT). X Yuan is a post-doctoral fellow supported by School of Medicine, Shandong University.

\section{Footnote}

Reporting Checklist: The authors have completed the Narrative Review reporting checklist. Available at http:// 


\section{Page 12 of 16}

dx.doi.org/10.21037/atm-20-5024

Conflicts of Interest: All authors have completed the ICMJE uniform disclosure form (available at http://dx.doi. org/10.21037/atm-20-5024). The authors have no conflicts of interest to declare.

Ethical Statement: The authors are accountable for all aspects of the work in ensuring that questions related to the accuracy or integrity of any part of the work are appropriately investigated and resolved.

Open Access Statement: This is an Open Access article distributed in accordance with the Creative Commons Attribution-NonCommercial-NoDerivs 4.0 International License (CC BY-NC-ND 4.0), which permits the noncommercial replication and distribution of the article with the strict proviso that no changes or edits are made and the original work is properly cited (including links to both the formal publication through the relevant DOI and the license). See: https://creativecommons.org/licenses/by-nc-nd/4.0/.

\section{References}

1. Wiltshire JJ, Drake TM, Uttley L, et al. Systematic Review of Trends in the Incidence Rates of Thyroid Cancer. Thyroid 2016;26:1541-52.

2. Wang J, Yu F, Shang Y, et al. Thyroid cancer: incidence and mortality trends in China, 2005-2015. Endocrine 2020;68:163-73.

3. Yan KL, Li S, Tseng CH, et al. Rising Incidence and Incidence-Based Mortality of Thyroid Cancer in California, 2000-2017. J Clin Endocrinol Metab 2020;105:dgaa121.

4. Asa SL. The Current Histologic Classification of Thyroid Cancer. Endocrinol Metab Clin North Am 2019;48:1-22.

5. Xing M. Molecular pathogenesis and mechanisms of thyroid cancer. Nat Rev Cancer 2013;13:184-99.

6. Network CGAR. Integrated genomic characterization of papillary thyroid carcinoma. Cell 2014;159:676-90.

7. Landa I, Ibrahimpasic T, Boucai L, et al. Genomic and transcriptomic hallmarks of poorly differentiated and anaplastic thyroid cancers. J Clin Invest 2016;126:1052-66.

8. Liang J, Cai W, Feng D, et al. Genetic landscape of papillary thyroid carcinoma in the Chinese population. J Pathol 2018;244:215-26.

9. Xing M. Genetic-guided Risk Assessment and Management of Thyroid Cancer. Endocrinol Metab Clin

\section{Yuan et al. TERT promoter mutations in thyroid carcinoma}

North Am 2019;48:109-24.

10. Oishi N, Kondo T, Ebina A, et al. Molecular alterations of coexisting thyroid papillary carcinoma and anaplastic carcinoma: identification of TERT mutation as an independent risk factor for transformation. Mod Pathol 2017;30:1527-37.

11. Hanahan D, Weinberg RA. Hallmarks of cancer: the next generation. Cell 2011;144:646-74.

12. Yuan $\mathrm{X}$, Larsson $\mathrm{C}, \mathrm{Xu} \mathrm{D}$. Mechanisms underlying the activation of TERT transcription and telomerase activity in human cancer: old actors and new players. Oncogene 2019;38:6172-83.

13. Yuan X, Dai M, Xu D. Telomere- Related Markers for Cancer. Curr Top Med Chem 2020;20:410-32.

14. Donati B, Ciarrocchi A. Telomerase and Telomeres Biology in Thyroid Cancer. Int J Mol Sci 2019;20:2887.

15. Zhang A, Wang J, Zheng B, et al. Telomere attrition predominantly occurs in precursor lesions during in vivo carcinogenic process of the uterine cervix. Oncogene 2004;23:7441-7.

16. Chiba K, Lorbeer FK, Shain AH, et al. Mutations in the promoter of the telomerase gene TERT contribute to tumorigenesis by a two-step mechanism. Science 2017;357:1416-20.

17. Liu T, Wang N, Cao J, et al. The age- and shorter telomere-dependent TERT promoter mutation in follicular thyroid cell-derived carcinomas. Oncogene 2014;33:4978-84.

18. Al-Suhaimi EA, Al-Khater K. Functions of stem cells of thyroid glands in health and disease. Rev Endocr Metab Disord 2019;20:187-95.

19. Vizioli MG, Possik PA, Tarantino E, et al. Evidence of oncogene-induced senescence in thyroid carcinogenesis. Endocr Relat Cancer 2011;18:743-57.

20. Zou M, Baitei EY, Al-Rijjal RA, et al. TSH overcomes Braf(V600E)-induced senescence to promote tumor progression via downregulation of $\mathrm{p} 53$ expression in papillary thyroid cancer. Oncogene 2016;35:1909-18.

21. Franco AT, Malaguarnera R, Refetoff S, et al. Thyrotrophin receptor signaling dependence of Brafinduced thyroid tumor initiation in mice. Proc Natl Acad Sci U S A 2011;108:1615-20.

22. Haymart MR, Glinberg SL, Liu J, et al. Higher serum TSH in thyroid cancer patients occurs independent of age and correlates with extrathyroidal extension. Clin Endocrinol (Oxf) 2009;71:434-9.

23. Yu S, Cao S, Hong S, et al. miR-3619-3p promotes papillary thyroid carcinoma progression via $\mathrm{Wnt} /$ beta- 
catenin pathway. Ann Transl Med 2019;7:643.

24. Wang J, Xie LY, Allan S, et al. Myc activates telomerase. Genes Dev 1998;12:1769-74.

25. Xu D, Popov N, Hou M, et al. Switch from Myc/Max to Mad1/Max binding and decrease in histone acetylation at the telomerase reverse transcriptase promoter during differentiation of HL60 cells. Proc Natl Acad Sci U S A 2001;98:3826-31.

26. Hoffmeyer K, Raggioli A, Rudloff S, et al. Wnt/betacatenin signaling regulates telomerase in stem cells and cancer cells. Science 2012;336:1549-54.

27. Zhang Y, Toh L, Lau P, et al. Human telomerase reverse transcriptase (hTERT) is a novel target of the Wnt/ beta-catenin pathway in human cancer. J Biol Chem 2012;287:32494-511.

28. Xu D, Wang Q, Gruber A, et al. Downregulation of telomerase reverse transcriptase mRNA expression by wild type p53 in human tumor cells. Oncogene 2000;19:5123-33.

29. Liu R, Xing M. TERT promoter mutations in thyroid cancer. Endocr Relat Cancer 2016;23:R143-55.

30. Liu T, Yuan X, Xu D. Cancer-Specific Telomerase Reverse Transcriptase (TERT) Promoter Mutations: Biological and Clinical Implications. Genes (Basel) 2016;7:38.

31. Huang FW, Hodis E, Xu MJ, et al. Highly recurrent TERT promoter mutations in human melanoma. Science 2013;339:957-9.

32. Horn S, Figl A, Rachakonda PS, et al. TERT promoter mutations in familial and sporadic melanoma. Science 2013;339:959-61.

33. Yuan X, Mu N, Wang N, et al. GABPA inhibits invasion/ metastasis in papillary thyroid carcinoma by regulating DICER1 expression. Oncogene 2019;38:965-79.

34. Liu X, Bishop J, Shan Y, et al. Highly prevalent TERT promoter mutations in aggressive thyroid cancers. Endocr Relat Cancer 2013;20:603-10.

35. Landa I, Ganly I, Chan TA, et al. Frequent somatic TERT promoter mutations in thyroid cancer: higher prevalence in advanced forms of the disease. J Clin Endocrinol Metab 2013;98:E1562-6.

36. Stenman A, Hysek M, Jatta K, et al. TERT Promoter Mutation Spatial Heterogeneity in a Metastatic Follicular Thyroid Carcinoma: Implications for Clinical Work-Up. Endocr Pathol 2019;30:246-8.

37. Argyropoulou M, Veskoukis AS, Karanatsiou PM, et al. Low Prevalence of TERT Promoter, BRAF and RAS Mutations in Papillary Thyroid Cancer in the Greek Population. Pathol Oncol Res 2020;26:347-54.
38. Ren H, Shen Y, Hu D, et al. Co-existence of BRAF(V600E) and TERT promoter mutations in papillary thyroid carcinoma is associated with tumor aggressiveness, but not with lymph node metastasis. Cancer Manag Res 2018;10:1005-13.

39. Yang J, Gong Y, Yan S, et al. Association between TERT promoter mutations and clinical behaviors in differentiated thyroid carcinoma: a systematic review and meta-analysis. Endocrine 2020;67:44-57.

40. Wang N, Liu T, Sofiadis A, et al. TERT promoter mutation as an early genetic event activating telomerase in follicular thyroid adenoma (FTA) and atypical FTA. Cancer 2014;120:2965-79.

41. Barthel FP, Wei W, Tang M, et al. Systematic analysis of telomere length and somatic alterations in 31 cancer types. Nat Genet 2017;49:349-57.

42. Bullock M, Lim G, Zhu Y, et al. ETS Factor ETV5 Activates the Mutant Telomerase Reverse Transcriptase Promoter in Thyroid Cancer. Thyroid 2019;29:1623-33.

43. Bell RJ, Rube HT, Kreig A, et al. Cancer. The transcription factor GABP selectively binds and activates the mutant TERT promoter in cancer. Science 2015;348:1036-9.

44. Stern JL, Theodorescu D, Vogelstein B, et al. Mutation of the TERT promoter, switch to active chromatin, and monoallelic TERT expression in multiple cancers. Genes Dev 2015;29:2219-24.

45. Liu R, Zhang T, Zhu G, et al. Regulation of mutant TERT by BRAF V600E/MAP kinase pathway through FOS/GABP in human cancer. Nat Commun 2018;9:579.

46. Guo Y, Yuan X, Li K, et al. GABPA is a master regulator of luminal identity and restrains aggressive diseases in bladder cancer. Cell Death Differ 2020;27:1862-77.

47. Yuan X, Dai M, Xu D. TERT promoter mutations and GABP transcription factors in carcinogenesis: More foes than friends. Cancer Lett 2020;493:1-9.

48. Rosmarin AG, Resendes KK, Yang Z, et al. GA-binding protein transcription factor: a review of GABP as an integrator of intracellular signaling and protein-protein interactions. Blood Cells Mol Dis 2004;32:143-54.

49. Mancini A, Xavier-Magalhaes A, Woods WS, et al. Disruption of the beta1L Isoform of GABP Reverses Glioblastoma Replicative Immortality in a TERT Promoter Mutation-Dependent Manner. Cancer Cell 2018;34:513-28.e8.

50. Zhang S, Zhang K, Ji P, et al. GABPA predicts prognosis and inhibits metastasis of hepatocellular carcinoma. BMC Cancer 2017;17:380.

51. DeLellis R. Pathology and Genetics of Tumours of 
Endocrine Organs. WHO Classification of Tumours. 3 ed., 2004.

52. Lloyd RV. WHO Classification of Tumours of Endocrine Organs. Fourth edition. In: Lloyd RV, Osamura RY, Klöppel G, et al. editors. WHO/IARC Classification of Tumours, 2017.

53. Hysek M, Paulsson JO, Jatta K, et al. Clinical Routine TERT Promoter Mutational Screening of Follicular Thyroid Tumors of Uncertain Malignant Potential (FTUMPs): A Useful Predictor of Metastatic Disease. Cancers (Basel) 2019;11:1443.

54. Hosen MI, Sheikh M, Zvereva M, et al. Urinary TERT promoter mutations are detectable up to 10 years prior to clinical diagnosis of bladder cancer: Evidence from the Golestan Cohort Study. EBioMedicine 2020;53:102643.

55. Chien MN, Yang PS, Hsu YC, et al. Transcriptome analysis of papillary thyroid cancer harboring telomerase reverse transcriptase promoter mutation. Head Neck 2018;40:2528-37.

56. Luo Z, Wang W, Li F, et al. Pan-cancer analysis identifies telomerase-associated signatures and cancer subtypes. Mol Cancer 2019;18:106.

57. Liu R, Bishop J, Zhu G, et al. Mortality Risk Stratification by Combining BRAF V600E and TERT Promoter Mutations in Papillary Thyroid Cancer: Genetic Duet of BRAF and TERT Promoter Mutations in Thyroid Cancer Mortality. JAMA Oncol 2017;3:202-8.

58. Xing M, Liu R, Liu X, et al. BRAF V600E and TERT promoter mutations cooperatively identify the most aggressive papillary thyroid cancer with highest recurrence. J Clin Oncol 2014;32:2718-26.

59. Yuan X, Cheng G, Yu J, et al. The TERT promoter mutation incidence is modified by germline TERT rs2736098 and rs2736100 polymorphisms in hepatocellular carcinoma. Oncotarget 2017;8:23120-9.

60. Dahlstrom J, Liu T, Yuan X, et al. TERT rs2736100 genotypes are associated with differential risk of myeloproliferative neoplasms in Swedish and Chinese male patient populations. Ann Hematol 2016;95:1825-32.

61. Maryoung L, Yue Y, Young A, et al. Somatic mutations in telomerase promoter counterbalance germline loss-offunction mutations. J Clin Invest 2017;127:982-6.

62. Kim W, Ludlow AT, Min J, et al. Regulation of the Human Telomerase Gene TERT by Telomere Position EffectOver Long Distances (TPE-OLD): Implications for Aging and Cancer. PLoS Biol 2016;14:e2000016.

63. Yuan X, Xu D. Telomerase Reverse Transcriptase (TERT) in Action: Cross-Talking with Epigenetics. Int J Mol Sci
2019;20:3338.

64. Yu J, Yuan X, Sjoholm L, et al. Telomerase reverse transcriptase regulates DNMT3B expression/aberrant DNA methylation phenotype and AKT activation in hepatocellular carcinoma. Cancer Lett 2018;434:33-41.

65. Liu Z, Li Q, Li K, et al. Telomerase reverse transcriptase promotes epithelial-mesenchymal transition and stem celllike traits in cancer cells. Oncogene 2013;32:4203-13.

66. Zhang K, Guo Y, Wang X, et al. WNT/beta-Catenin Directs Self-Renewal Symmetric Cell Division of hTERT(high) Prostate Cancer Stem Cells. Cancer Res 2017;77:2534-47.

67. Bu R, Siraj AK, Divya SP, et al. Telomerase reverse transcriptase mutations are independent predictor of disease-free survival in Middle Eastern papillary thyroid cancer. Int J Cancer 2018;142:2028-39.

68. Zhang X, Li B, Yu J, et al. MYC-dependent downregulation of telomerase by FLT3 inhibitors is required for their therapeutic efficacy on acute myeloid leukemia. Ann Hematol 2018;97:63-72.

69. Ci X, Li B, Ma X, et al. Bortezomib-mediated downregulation of telomerase and disruption of telomere homeostasis contributes to apoptosis of malignant cells. Oncotarget 2015;6:38079-92.

70. Liu R, Xing M. Diagnostic and prognostic TERT promoter mutations in thyroid fine-needle aspiration biopsy. Endocr Relat Cancer 2014;21:825-30.

71. Censi S, Cavedon E, Bertazza L, et al. Frequency and Significance of Ras, Tert Promoter, and Braf Mutations in Cytologically Indeterminate Thyroid Nodules: A Monocentric Case Series at a Tertiary-Level Endocrinology Unit. Front Endocrinol (Lausanne) 2017;8:273.

72. Decaussin-Petrucci M, Descotes F, Depaepe L, et al. Molecular testing of BRAF, RAS and TERT on thyroid FNAs with indeterminate cytology improves diagnostic accuracy. Cytopathology 2017;28:482-7.

73. Giorgenon TMV, Carrijo FT, Arruda MA, et al. Preoperative detection of TERT promoter and BRAFV600E mutations in papillary thyroid carcinoma in high-risk thyroid nodules. Arch Endocrinol Metab 2019;63:107-12.

74. Crescenzi A, Trimboli P, Modica DC, et al. Preoperative Assessment of TERT Promoter Mutation on Thyroid Core Needle Biopsies Supports Diagnosis of Malignancy and Addresses Surgical Strategy. Horm Metab Res 2016;48:157-62.

75. Shi H, Guo LH, Zhang YF, et al. Suspicious ultrasound 
and clinicopathological features of papillary thyroid carcinoma predict the status of TERT promoter. Endocrine 2020;68:349-57.

76. Hahn SY, Kim TH, Ki CS, et al. Ultrasound and clinicopathological features of papillary thyroid carcinomas with BRAF and TERT promoter mutations. Oncotarget 2017;8:108946-57.

77. Kim TH, Ki CS, Hahn SY, et al. Ultrasonographic prediction of highly aggressive telomerase reverse transcriptase (TERT) promoter-mutated papillary thyroid cancer. Endocrine 2017;57:234-40.

78. Zhu Y, Sang Q, Jia S, et al. Deep neural networks could differentiate Bethesda class III versus class IV/V/VI. Ann Transl Med 2019;7:231.

79. Wang K, Liu T, Ge N, et al. TERT promoter mutations are associated with distant metastases in upper tract urothelial carcinomas and serve as urinary biomarkers detected by a sensitive castPCR. Oncotarget 2014;5:12428-39.

80. Tanaka A, Matsuse M, Saenko V, et al. TERT mRNA Expression as a Novel Prognostic Marker in Papillary Thyroid Carcinomas. Thyroid 2019;29:1105-14.

81. Nikiforov YE, Carty SE, Chiosea SI, et al. Highly accurate diagnosis of cancer in thyroid nodules with follicular neoplasm/suspicious for a follicular neoplasm cytology by ThyroSeq v2 next-generation sequencing assay. Cancer 2014;120:3627-34.

82. Yu FX, Hu MX, Zhao HX, et al. Precise Detection of Gene Mutations in Fine-Needle Aspiration Specimens of the Papillary Thyroid Microcarcinoma Using Next-Generation Sequencing. Int J Endocrinol 2019;2019:4723958.

83. Colombo C, Muzza M, Proverbio MC, et al. Impact of Mutation Density and Heterogeneity on Papillary Thyroid Cancer Clinical Features and Remission Probability. Thyroid 2019;29:237-51.

84. Censi S, Barollo S, Grespan E, et al. Prognostic significance of TERT promoter and BRAF mutations in TIR-4 and TIR-5 thyroid cytology. Eur J Endocrinol 2019;181:1-11.

85. Jin A, Xu J, Wang Y. The role of TERT promoter mutations in postoperative and preoperative diagnosis and prognosis in thyroid cancer. Medicine (Baltimore) 2018;97:e11548.

86. Xing X, Mu N, Yuan X, et al. PLEKHS1 Over-Expression Is Associated with Metastases and Poor Outcomes in Papillary Thyroid Carcinoma. Cancers (Basel) 2020;12:2133.
87. Melo M, Gaspar da Rocha A, Batista R, et al. TERT, BRAF, and NRAS in Primary Thyroid Cancer and Metastatic Disease. J Clin Endocrinol Metab 2017;102:1898-907.

88. Vuong HG, Altibi AM, Duong UN, et al. Role of molecular markers to predict distant metastasis in papillary thyroid carcinoma: Promising value of TERT promoter mutations and insignificant role of BRAF mutations-a meta-analysis. Tumour Biol 2017;39:1010428317713913.

89. Kim TH, Ki CS, Kim HS, et al. Refining Dynamic Risk Stratification and Prognostic Groups for Differentiated Thyroid Cancer With TERT Promoter Mutations. J Clin Endocrinol Metab 2017;102:1757-64.

90. Shen X, Liu R, Xing M. A six-genotype genetic prognostic model for papillary thyroid cancer. Endocr Relat Cancer 2017;24:41-52.

91. Jin L, Chen E, Dong S, et al. BRAF and TERT promoter mutations in the aggressiveness of papillary thyroid carcinoma: a study of 653 patients. Oncotarget 2016;7:18346-55

92. Bae JS, Kim Y, Jeon S, et al. Clinical utility of TERT promoter mutations and ALK rearrangement in thyroid cancer patients with a high prevalence of the BRAF V600E mutation. Diagn Pathol 2016;11:21.

93. Sun J, Zhang J, Lu J, et al. BRAF V600E and TERT Promoter Mutations in Papillary Thyroid Carcinoma in Chinese Patients. PLoS One 2016;11:e0153319.

94. Melo M, da Rocha AG, Vinagre J, et al. TERT promoter mutations are a major indicator of poor outcome in differentiated thyroid carcinomas. J Clin Endocrinol Metab 2014;99:E754-65.

95. Penna GC, Pestana A, Cameselle JM, et al. TERTp mutation is associated with a shorter progression free survival in patients with aggressive histology subtypes of follicular-cell derived thyroid carcinoma. Endocrine 2018;61:489-98.

96. Nasirden A, Saito T, Fukumura Y, et al. In Japanese patients with papillary thyroid carcinoma, TERT promoter mutation is associated with poor prognosis, in contrast to BRAF (V600E) mutation. Virchows Arch 2016;469:687-96.

97. Lee SE, Hwang TS, Choi YL, et al. Prognostic Significance of TERT Promoter Mutations in Papillary Thyroid Carcinomas in a BRAF(V600E) MutationPrevalent Population. Thyroid 2016;26:901-10.

98. Song YS, Lim JA, Choi H, et al. Prognostic effects of TERT promoter mutations are enhanced by coexistence with BRAF or RAS mutations and strengthen the 


\section{Page 16 of 16}

risk prediction by the ATA or TNM staging system in differentiated thyroid cancer patients. Cancer 2016;122:1370-9.

99. Malik N, Nikitski AV, Klam E, et al. Molecular Profile and Clinical Outcomes in Differentiated Thyroid Cancer Patients Presenting with Bone Metastasis. Endocr Pract 2019;25:1255-62.

100. Yang X, Li J, Li X, et al. TERT Promoter Mutation Predicts Radioiodine-Refractory Character in Distant Metastatic Differentiated Thyroid Cancer. J Nucl Med 2017;58:258-65.

101.Liu J, Liu R, Shen X, et al. The Genetic Duet of BRAF V600E and TERT Promoter Mutations Robustly Predicts Loss of Radioiodine Avidity in Recurrent Papillary Thyroid Cancer. J Nucl Med 2020;61:177-82.

102. Tan J, Liu R, Zhu G, et al. TERT promoter mutation determines apoptotic and therapeutic responses of BRAFmutant cancers to BRAF and MEK inhibitors: Achilles Heel. Proc Natl Acad Sci U S A 2020;117:15846-51.

103.Li X, Qian X, Wang B, et al. Programmable base editing of mutated TERT promoter inhibits brain tumour growth.

\section{Yuan et al. TERT promoter mutations in thyroid carcinoma}

Nat Cell Biol 2020;22:282-8.

104. Lin S, Shen J, Zhao W, et al. Evaluating the effectiveness of targeted therapies for thyroid carcinoma: an updated meta-analysis. Ann Transl Med 2019;7:802.

105.Lombardo GE, Maggisano V, Celano M, et al. AntihTERT siRNA-Loaded Nanoparticles Block the Growth of Anaplastic Thyroid Cancer Xenograft. Mol Cancer Ther 2018;17:1187-95.

106. Dahlstrom J, Xia C, Xing X, et al. JAK2 inhibition in JAK2(V617F)-bearing leukemia cells enriches CD34(+) leukemic stem cells that are abolished by the telomerase inhibitor GRN163L. Biochem Biophys Res Commun 2020;527:425-31.

107. Tefferi A. Telomerase Inhibitor Imetelstat in Essential Thrombocythemia and Myelofibrosis. N Engl J Med 2015;373:2580-1.

108. Baerlocher GM, Burington B, Snyder DS. Telomerase Inhibitor Imetelstat in Essential Thrombocythemia and Myelofibrosis. N Engl J Med 2015;373:2580.

(English Language Editor: J. Reynolds)
Cite this article as: Yuan X, Liu T, Xu D. Telomerase reverse transcriptase promoter mutations in thyroid carcinomas: implications in precision oncology. Ann Transl Med 2020;8(19):1244. doi: 10.21037/atm-20-5024 